\title{
Fluorine-18 Fluorodeoxyglucose Positron Emission Tomography for Osteochondromas Utilizing a Triple-Time Point Protocol
}

\author{
Chris Sambaziotis ${ }^{1}$, Andrew Lovy ${ }^{1}$, Renee M. Moadel ${ }^{2}$, Murthy Chamarthy², Joseph Glaser ${ }^{2}$, \\ Srividya Jaini ${ }^{2}$, Esperanza Villanueva-Siles ${ }^{3}$, David S. Geller ${ }^{{ }^{*}}$ \\ ${ }^{1}$ Department of Orthopaedic Surgery, Montefiore Medical Center, Albert Einstein College of Medicine, New York, USA \\ ${ }^{2}$ Department of Nuclear Medicine, Montefiore Medical Center, Albert Einstein College of Medicine, New York, USA \\ ${ }^{3}$ Department of Pathology, Montefiore Medical Center, Albert Einstein College of Medicine, New York, USA \\ E-mail:"dgeller@montefiore.org \\ Received October 11, 2011; revised December 1, 2011; accepted December 10, 2011
}

\begin{abstract}
Purpose: The purpose of this study was to assess solitary osteochondroma and hereditary multiple osteochondral exostoses (HMOCE) utilizing FDG PET and a triple time point protocol. Methods: Seven patients were consented and recruited for PET evaluation of presumed benign osteochondroma. Following injection of $15 \mathrm{mCi}$ of FDG, the lesion(s) of interest was imaged with PET-CT at 45 minutes post injection, whole body at 50 minutes post, and lesion of interest at 95 minutes post injection. A maximum standardized uptake value $\left(\mathrm{SUV}_{\max }\right)$ was obtained for the lesion(s) of interest at each time point, and an $\mathrm{SUV}_{\Delta}$ was calculated for each lesion of interest from the first time point to the third time point. Results: 16 lesions from 7 patients were included in the study. Mean $\mathrm{SUV}_{\max }$ for all 3 time points was 1.04 with a standard deviation of 0.50 (range $0.3-2.2$ ). The mean SUV was 0.096 with a range of 0 - 0.4. Among the 3 patients with histologically confirmed osteochondromas, mean $\mathrm{SUV}_{\max }$ was 0.67 , with standard deviation of 0.23 and range of 0.3 to 1.0. The mean $\mathrm{SUV}_{\Delta 13}$ was 0.081 (range $\left.0-0.4\right)$, mean $\mathrm{SUV}_{\Delta 12}$ was 0.10 (0 - 0.3), and mean $\mathrm{SUV}_{\Delta 23}$ was 0.11 (range 0 - 0.4) $(\mathrm{p}=0.74)$. Conclusion: Benign lesions were found to not have progressively increasing uptake on multiple time point FDG PET. Until chondrosarcomas are evaluated using triple time point ${ }^{18} \mathrm{FDG}$ PET, its applicability in the evaluation of osteochondroma versus malignant change remains uncertain.
\end{abstract}

Keywords: Positron Emission Tomography, Osteochondroma, Chondrosarcoma, Fluorine-18 Fluorodeoxyglucose

\section{Introduction}

Osteochondromas are the most common benign cartilagenous tumors, typically presenting during the second decade and often diagnosed as incidental radiological findings [1]. Lesions may be solitary or multiple as in hereditary multiple osteochondral exostoses (HMOCE), which is an autosomal dominant disorder characterized by two or more exostoses. The primary concern for patients with osteochondromas or HMOCE is the risk of malignant transformation, with described rates of less than $1 \%$ and $5 \%$ to $25 \%$ respectively [2-4]. Asymptomatic lesions require observation and can be closely monitored for signs of malignant change.
The key to surveillance is early detection of malignant transformation; however this is a daunting task especially in HMOCE, where multiple lesions limits conventional imaging modalities and continued growth beyond skeletal maturity may occur $[5,6]$. While several imaging modalities including radiographs, ultrasound, magnetic resonance imaging (MRI) and computed tomography (CT) are used for initial evaluation, F18 fluoro-2-deoxy-glucose $\left({ }^{18} \mathrm{FDG}\right)$ positron emission tomography (PET) can provide additional physiologic information. ${ }^{18} \mathrm{FDG}$ shares an intracellular transport mechanism with glucose, resulting in increased uptake in metabolically active cells [7].

As a result, ${ }^{18}$ FDG PET has become increasingly re- 
lied upon for diagnosis and staging of numerous neoplastic processes including lung, breast, and colon cancer [7]. Several studies have also evaluated the diagnostic value of PET in a variety of musculoskeletal lesions [3,5,8-10], and have reportedly distinguished benign from malignant musculoskeletal lesions with a sensitivity of $90.9 \%$ to $91.7 \%$ and specificity of $100 \%$ in trials involving 29 and 45 patients respectively $[11,12]$. The diagnostic criteria of these studies are largely based upon standardized uptake values (SUV) obtained at a single time point of 60 minutes following injection of ${ }^{18} \mathrm{FDG}$, despite ${ }^{18} \mathrm{FDG}$ 's half-life of approximately 110 minutes. Additional studies have evaluated the use of dual time point ${ }^{18} \mathrm{FDG}$ PET, noting additional diagnostic accuracy compared to standard single time point ${ }^{18}$ FDG PET scanning $[13,14]$. The purpose of this study is to assess and describe the inherent characteristics of ${ }^{18}$ FDG PET utilizing triple time points in solitary osteochondroma and HMOCE. We hypothesize that the addition of a third time point will result in better characterization of osteochondromas based on the half-life of ${ }^{18} \mathrm{FDG}$.

\section{Methods}

Approval was obtained from the Institutional Review Board prior to beginning of the study. From 2008 to 2010 , all patients with suspected or diagnosed osteochondroma or chondrosarcoma, including patients with recurrence, were eligible for participation in the trial. Pregnant and/or lactating women, infants and children under 12 years of age and those subjects who would not be able to tolerate the exam were excluded from the study. In addition, diabetic patients were also excluded to prevent interference with quantitation of FDG metabolism. Patients participating in the study were scheduled for an FDG PET-CT scan utilizing a triple time point protocol.

Following history, physical exam, and conventional imaging studies such as plain radiographs, MRI or CT, patients underwent a triple time point FDG PET-CT scan. Before undergoing FDG PET-CT study, patients were instructed to fast for 4 hours prior to the scan and blood sugar levels were checked to ensure fasting levels of $<160 \mathrm{mg} / \mathrm{dL}$. Intravenous access was obtained and 15 $\mathrm{mCi}$ of FDG was injected intravenously in standard fashion. Low dose CT scans were performed using 120 $\mathrm{kV}$ and $50-100 \mathrm{mAs}$ for adults and $20-30 \mathrm{mAs}$ for children. Field-Of-View (FOV) was $600 \mathrm{~mm}$, with $5 \mathrm{~mm}$ slices acquired in increments of 5. Collimation was separate on all scanners. Pitch and rotation were 0.813 and 0.5 , respectively. Imaging matrix was $512 \times 512$. After injection, three PET-CT scans were obtained. The first scan was a regional scan that started approximately
45 minutes $\left(T_{1}\right)$ post injection and proceeded for 5 minutes over the tumor(s) of interest. The second scan was a whole body scan (top of head to toes) and started approximately 50 minutes $\left(\mathrm{T}_{2}\right)$ post injection and proceeded for approximately 30 minutes. The third scan was a regional scan that started approximately 95 minutes $\left(T_{3}\right)$ post injection and proceeded for 5 minutes over the tumor(s) of interest. All images were acquired using a Philips GEMINI TF PET-CT system (Netherlands) and reconstructed utilizing an iterative reconstruction algorithm with low-dose CT attenuation correction. Images were analyzed on a Philips workstation in the transaxial, coronal and sagittal planes. A maximum standardized uptake value $\left(\mathrm{SUV}_{\max }\right)$ was obtained for the tumor(s) of interest within the field of view at each time point (SU$\mathrm{V}_{\max } \mathrm{T}_{1}, \mathrm{SUV}_{\max } \mathrm{T}_{2}, \mathrm{SUV}_{\max } \mathrm{T}_{3}$ ) using the equation below.

$$
\begin{aligned}
\mathrm{SUV}= & \text { tissue activity }(\mathrm{mCi} / \mathrm{mg}) /[\text { injected FDG dose } \\
& (\mathrm{mCi}) / \text { body weight }(\mathrm{kg})]
\end{aligned}
$$

Additionally, SUV deltas $\left(\mathrm{SUV}_{\Delta 12}=\mathrm{SUV}_{\max } \mathrm{T}_{1}-\right.$ $\mathrm{SUV}_{\max } \mathrm{T}_{2}, \mathrm{SUV}_{\Delta 13}=\mathrm{SUV}_{\max } \mathrm{T}_{1}-\mathrm{SUV}_{\max } \mathrm{T}_{3}, \mathrm{SUV}_{\Delta 23}=$ $\mathrm{SUV}_{\max } \mathrm{T}_{2}-\mathrm{SUV}_{\max } \mathrm{T}_{3}$ ) were calculated for the tumor(s) of interest. A SUV $\mathrm{Sax}_{\max }$ was measured for all other tumors seen in the whole body scan at $\mathrm{T}_{2}$.

When possible, diagnosis was confirmed histologically. In the majority of cases osteochondromas exhibit normal-appearing medullary bone and marrow fat/elements surrounded peripherally, by a variable thickened cartilagious cap. The chondrocytes in the cartilage may be solitary or clustered and may undergo enchondral ossification resembling the epiphyseal growth plate.

The mean, range and standard deviation of the $\mathrm{SUV}_{\max }$ and SUV measurements were calculated for both osteochondromas and HMOCE lesions, and compared using Wilcoxon rank sum test, and Kruskal-Wallis test.

\section{Results}

Seven patients with a total of 16 discrete lesions were enrolled in the study, of which 3 patients had solitary osteochondromas and 4 patients had HMOCE (Table 1, Figure 1). The mean $\mathrm{SUV}_{\max }$ from all 3 time points was 1.04 with a standard deviation of 0.50 and range of 0.3 2.2 (Table 2). The mean $\operatorname{SUV}_{\Delta 13}$, was 0.081 with a range of $0-0.4$, the mean $\mathrm{SUV}_{\Delta 12}$ was 0.10 with a range of $0-0.3$, and the mean $\mathrm{SUV}_{\triangle 23}$. was 0.11 with a range of $0-0.4(p=0.74)$. Among patients with HMOCE, 3 patients had 3 lesions and 1 patient had 4 lesions for a total of 13 lesions in the study. There was no difference in mean $\mathrm{SUV}_{\max }$ among patients with solitary lesions $(0.96 \pm 0.12)$ compared to patients with HMOCE $(1.06 \pm$ $0.55)(\mathrm{p}=0.68)$. The mean $\mathrm{SUV}_{\Delta}$ among patient with solitary lesions and HMOCE was 0.022 and 0.11 respec- 
Table 1. Patient demographic characteristics.

\begin{tabular}{lc}
\hline Age: $y r s$ & $35.1 \pm 9.8$ \\
\hline Total: $\mathrm{n}$ & 7 \\
Male: $\mathrm{n}(\%)$ & $4(57 \%)$ \\
HMOCE: $\mathrm{n}(\%)$ & $4(57 \%)$ \\
Solitary Osteochondroma: $\mathrm{n}(\%)$ & $3(43 \%)$ \\
\hline
\end{tabular}

HMOCE $=$ hereditary multiple osteochondral exostoses.

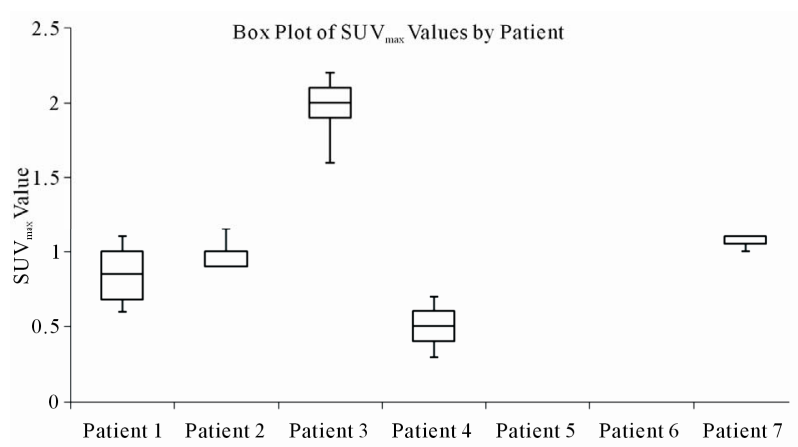

Figure 1. Box plot of $\mathrm{SUV}_{\max }$ values by patient.

tively $(\mathrm{p}=0.02)$.

Tumors were histologically confirmed to be benign in 3 patients who underwent surgical resection secondary to pain (Figures 2 and 3). The mean $\mathrm{SUV}_{\max }$ among patients with confirmed benign lesions was 0.67 , with standard deviation of 0.23 and range of 0.3 to 1.0 .

Of note, patient 3 had an $\mathrm{SUV} \geq 2$ with a range of 1.6 to 2.2 , and there is no histopathological correlation. However, the patient remained clinically stable during 2-years follow-up.

\section{Discussion}

While advanced or large malignancies are readily identified using conventional radiographic methods, the early detection of malignant transformation of osteochondromas remains a clinical dilemma. Although historically a cartilage cap of $>1 \mathrm{~cm}$ was thought to indicate malignant potential, large benign caps have been described particularly in patients with HMOCE whose lesions may continue to grow beyond skeletal maturity. Bone scans are nonspecific and their ability to distinguish malignant from benign entities is poor. Previous studies have evaluated the diagnostic utility of positron emission tomography in musculoskeletal lesions, as well as in lung, breast, and colon cancer [3,5,7-10]. These studies suggested malignant transformation with maximal standardized uptake values greater than 2.0 or 3.0 , while more recent studies noted improved diagnostic accuracy util-

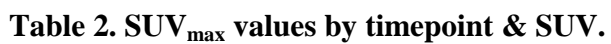

\begin{tabular}{|c|c|c|c|c|c|}
\hline & Time & Lesion 1 & Lesion 2 & Lesion 3 & Lesion 4 \\
\hline \multirow{4}{*}{ Patient 1} & $\mathrm{~T}_{1}$ & 1 & 0.6 & 1.1 & 0.8 \\
\hline & $\mathrm{T}_{2}$ & 0.9 & 0.6 & 1 & 0.8 \\
\hline & $\mathrm{T}_{3}$ & 0.9 & 0.6 & 1 & 0.7 \\
\hline & $\mathrm{SUV}_{\Delta 13}$ & 0.1 & 0 & 0.1 & 0.1 \\
\hline \multirow{4}{*}{ Patient 2} & $\mathrm{~T}_{1}$ & 1 & 0.9 & 1.2 & \\
\hline & $\mathrm{T}_{2}$ & 0.9 & 1 & 1 & \\
\hline & $\mathrm{T}_{3}$ & 1 & 0.9 & 1.2 & \\
\hline & $\mathrm{SUV}_{\Delta 13}$ & 0 & 0 & 0 & \\
\hline \multirow{4}{*}{ Patient 3} & $\mathrm{~T}_{1}$ & 2.1 & 1.9 & 1.8 & \\
\hline & $\mathrm{T}_{2}$ & 2.1 & 2.2 & 1.6 & \\
\hline & $\mathrm{T}_{3}$ & 2 & 1.9 & 2 & \\
\hline & $\mathrm{SUV}_{\Delta 13}$ & 0.1 & 0 & 0.2 & \\
\hline \multirow{4}{*}{ Patient $4 *$} & $\mathrm{~T}_{1}$ & 0.6 & 0.7 & 0.5 & \\
\hline & $\mathrm{T}_{2}$ & 0.7 & 0.5 & 0.4 & \\
\hline & $\mathrm{T}_{3}$ & 0.5 & 0.3 & 0.4 & \\
\hline & $\mathrm{SUV}_{\Delta 13}$ & 0.1 & 0.4 & 0.1 & \\
\hline \multirow{4}{*}{ Patient $5^{*}$} & $\mathrm{~T}_{1}$ & 0.8 & & & \\
\hline & $\mathrm{T}_{2}$ & 0.8 & & & \\
\hline & $\mathrm{T}_{3}$ & 0.8 & & & \\
\hline & $\mathrm{SUV}_{\Delta 13}$ & 0 & & & \\
\hline \multirow{4}{*}{ Patient $6^{*}$} & $\mathrm{~T}_{1}$ & 1 & & & \\
\hline & $\mathrm{T}_{2}$ & 1 & & & \\
\hline & $\mathrm{T}_{3}$ & 1 & & & \\
\hline & $\mathrm{SUV}_{\Delta 13}$ & 0 & & & \\
\hline \multirow{4}{*}{ Patient 7} & $\mathrm{~T}_{1}$ & 1 & & & \\
\hline & $\mathrm{T}_{2}$ & 1.1 & & & \\
\hline & $\mathrm{T}_{3}$ & 1.1 & & & \\
\hline & $\mathrm{SUV}_{\Delta 13}$ & 0.1 & & & \\
\hline
\end{tabular}

*Tumors were histologically confirmed to be benign. Only the $\mathrm{SUV}_{\Delta 13}$ is listed for each lesion due to the non-significant difference between the $\mathrm{SUV}_{\Delta 13}$ values.

izing dual time point PET scans compared to single time point scanning $[13,14]$.

Aoki et al., investigated the results of PET scan between benign cartilaginous tumors and chondrosarcoma and found that the average $\mathrm{SUV}_{\max }$ for the chondrosarcoma group to be $2.23 \pm 0.80$, with a range from 1.3 to 3.3 [8]. Based on these findings, a SUV of 1.3 was suggested as a possible cutoff for differentiating benign from malignant cartilaginous tumors. Similarly, Feldman et al. [11] found that an average $\mathrm{SUV}_{\max }$ of 2.0 differentiated between benign and malignant lesions with a sen- 


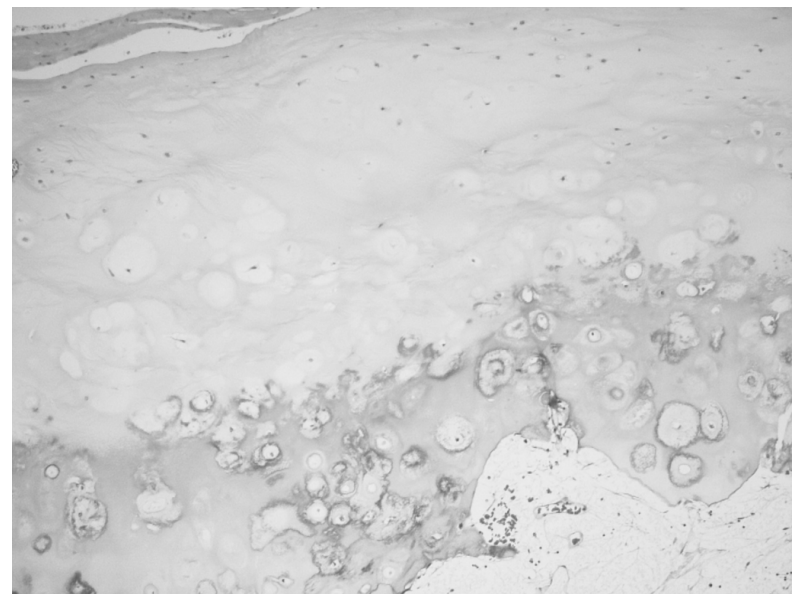

(a)

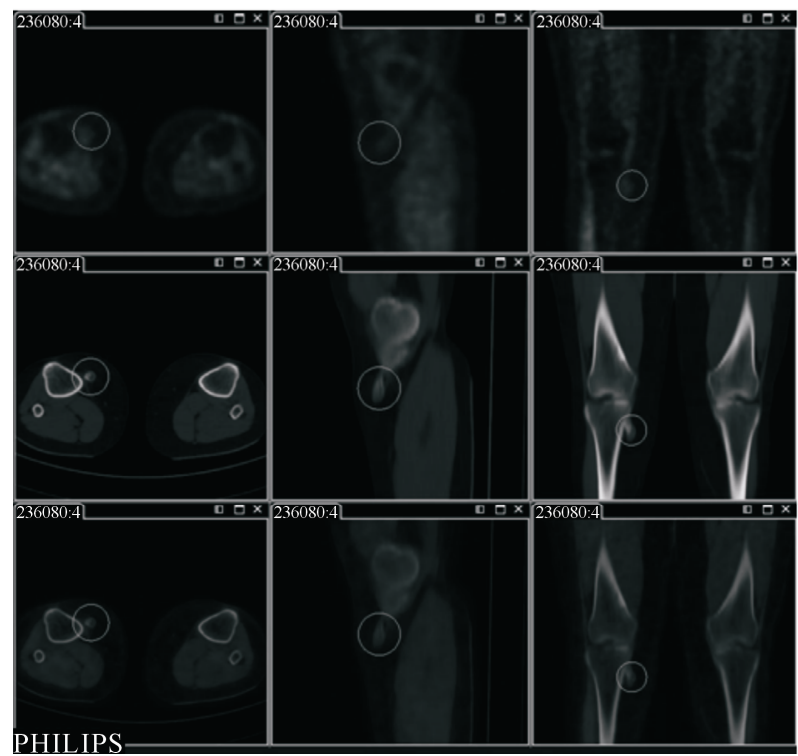

(b)

Figure 2. 19-year-old female with solitary right medial tibia osteochondroma that was surgically resected due to pain. (a) Osteochondroma on hematoxylin and eosin stain (20×) demonstrating calcification of the cartilage cap, before going enchondral ossification; (b) PET (first row), CT (second row) and fused (third row) displayed in the transanxial, sagittal and coronal planes showing the right medial tibia osteochondroma (circle) at $\mathrm{T}_{2}$ where $\mathrm{SUV}_{\max }=\mathbf{0 . 8}$.

sitivity of $91.7 \%$ and a specificity of $100 \%$. Additionally, all aggressive lesions had a $\mathrm{SUV}_{\max }>2.0$. More recent literature investigated the mean maximal standard uptake values in benign cartilaginous tumors, grade-1 chondrosarcomas, and high-grade chondrosarcomas [10]. Their results did not show a significant difference between benign cartilage tumors $(1.147 \pm 0.751)$, and grade- 1 chondrosarcomas $(0.898 \pm 0.908)$, but did show a significant difference between low-grade (benign and grade-1 chondrosarcoma) and high-grade chondrosarcomas

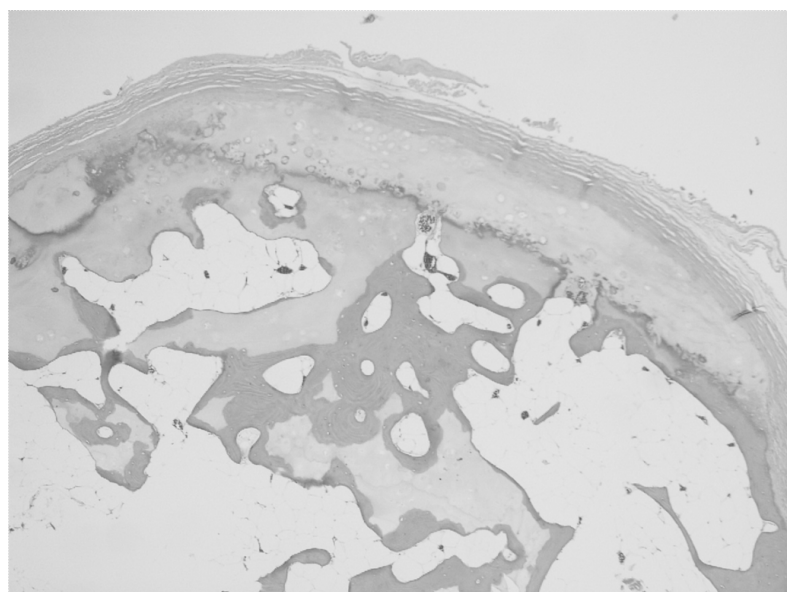

(a)

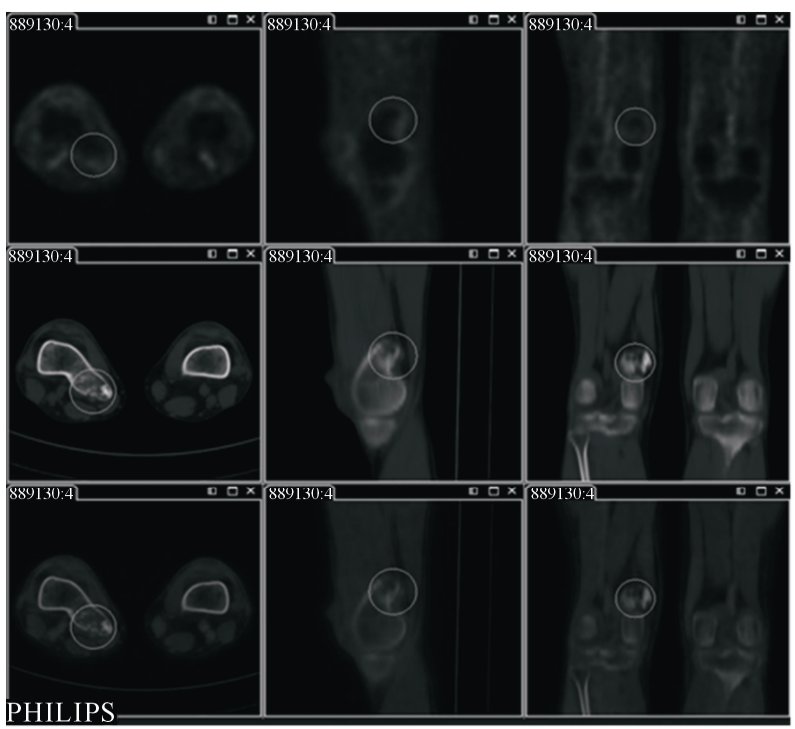

(b)

Figure 3. 25-year-old man with single right medial femur osteochondroma that was surgically resected due to pain. (a) Osteochondroma on hematoxylin and eosin stain $(10 \times)$ with hyaline cartilage cap covered by fibrous perichondrium. The superficial cancellous bone of the stalk is undergoing enchondral ossification; (b) PET (first row), CT (second row) and fused (third row) displayed in the transanxial, sagittal and coronal planes showing the right medial femur osteochondroma (circle) at $T_{2}$ where $S U V_{\max }=1.0$.

$(6.903 \pm 5.581)$. These results demonstrate the difficulty and radiological limitations in determining malignant transformation of benign cartilaginous tumors from low-grade chondrosarcomas.

The current study demonstrates concordant results with those previously published in regards to maximum standardized uptake values in benign osteochondromas. The previous cutoff of 2.0 is supported in our study when examining the $\mathrm{SUV}_{\max }$ in those with confirmed histologic benign lesions, with a range from 0.3 to 1.1 (mean $0.67 \pm$ 
0.24). Furthermore, our study demonstrates that benign lesions do not have progressively increasing uptake on multiple time point FDG PET unlike metabolically active malignant lesions as shown in the prior studies. Thus, for benign osteochondromas, the addition of a triple time point did not provide additional clinical utility.

The use of imaging modalities such as ultrasound, CT or MRI to differentiate benign osteochondromas from secondary chondrosarcomas is well described in the literature [15-18]. Cartilage cap thickness is commonly used as a marker of malignant transformation as malignant transformation typically occurs with cartilage cap thicknesses greater than $1-3 \mathrm{~cm}$ [15]. In a study of 101 patients, 34 of which had secondary chondrosarcomas, Bernard et al. [19] suggested a $2 \mathrm{~cm}$ cutoff based on their described imaging technique to distinguish benign osteochondromas from secondary chondrosarcomas. The sensitivity and specificity of a $2 \mathrm{~cm}$ cutoff was $100 \%$ and $98 \%$ for MRI and $100 \%$ and $95 \%$ for CT, which are encouraging results and which arguably may support this method of evaluation as the current standard of care [19].

Limitations of our study include a small sample size as well as a lack of malignant tumors for comparison and characterization. Even though maximum standardized uptake values utilizing triple time points were similar in histologically confirmed benign lesions, we were unable to make a similar conclusion for low or high-grade chondrosarcomas, which are inherently more metabolically active. In addition, the natural history of osteochondromas and their malignant transformation rate is quite rare, making it difficult to evaluate the use of PET scan to differentiate benign osteochondroma from chondrosarcoma at a single institution.

In conclusion, our preliminary study demonstrated that maximum standardized uptake values utilizing triple time point FDG PET for histology confirmed osteochondromas showed no difference to previously published values utilizing single or dual time point protocols. The clinical utility of triple time point protocol based on the half-life of FDG in chondrosarcomas, which are inherently more metabolically active, remains unknown. A multi-institutional study would provide increased numbers and the ability to detect the rare transformation of osteochondromas to chondrosarcomas. This may ultimately enable investigators to determine reliable and relevant SUV cutoff points allowing for the distinction of benign osteochondromas from low-grade chondrosarcomas.

\section{References}

[1] J. Biermann, "Common Benign Lesions of Bone in Children and Adolescents," Journal of Pediatric Orthopae- dics, Vol. 22, No. 2, 2002, pp. 268-273. doi:10.1097/01241398-200203000-00028

[2] R. C. Garrison, K. K. Unni, R. A. McLeod, D. J. Pritchard and D. C. Dahlin, "Chondrosarcoma Arising in Osteochondroma," Cancer, Vol. 49, No. 9, 1982, pp. 18901897.

doi:10.1002/1097-0142(19820501)49:9<1890::AID-CNC R2820490923>3.0.CO;2-U

[3] E. L. Staals, P. Bacchini, M. Mercuri and F. Bertoni, "Dedifferentiated Chondrosarcomas Arising in Preexisting Osteochondromas," Journal of Bone and Joint Surgery, Vol. 89, No. 5, 2007, pp. 987-993. doi:10.2106/JBJS.F.00288

[4] S. Pannier and L. Legeai-Mallet, "Hereditary Multiple Exostoses and Enchondromatosis," Best Practice \& Research Clinical Rheumatology, Vol. 22, No. 1, 2008, pp. 45-54. doi:10.1016/j.berh.2007.12.004

[5] F. Feldman, R. Vanheertum and C. Saxena, "18Fluorodeoxyglucose Positron Emission Tomography Evaluation of Benign versus Malignant Osteochondromas: Preliminary Observations," Journal of Computer Assisted Tomography, Vol. 30, No. 5, 2006, pp. 858-864.

doi:10.1097/01.rct.0000228160.86096.ca

[6] A. Kivioja, H. Ervasti, J. Kinnunen, I. Kaitila, M. Wolf and T. Bohling, "Chondrosarcoma in a Family with Multiple Hereditary Exostoses," Journal of Bone and Joint Surgery, Vol. 82, No. 2, 2000, pp. 261-266. doi:10.1302/0301-620X.82B2 .10139

[7] D. A. Podoloff, D. W. Ball, E. Ben-Josef, et al., "NCCN Task Force: Clinical Utility of PET in a Variety of Tumor Types," Journal of the National Comprehensive Cancer Network, Vol. 7, Suppl. 2, 2009, pp. S1-S26.

[8] J. Aoki, H. Watanabe, T. Shinozaki, M. Tokunaga, T. Inoue and K. Endo, "FDG-PET in Differential Diagnosis and Grading of Chondrosarcomas," Journal of Computer Assisted Tomography, Vol. 23, No. 4, 1999, pp. 603 -608. doi:10.1097/00004728-199907000-00022

[9] E. U. Conrad, H. D. Morgan, C. Vernon, S. M. Schuetze and J. F. Eary, "Fluorodeoxyglucose Positron Emission Tomography Scanning: Basic Principles and Imaging of Adult Soft-Tissue Sarcomas," Journal of Bone and Joint Surgery, Vol. 86-A, No. 2, 2004, pp. 98-104.

[10] F. Y. Lee, J. Yu, S. S. Chang, R. Fawwaz and M. V. Parisien, "Diagnostic Value and Limitations of Fluorine-18 Fluorodeoxyglucose Positron Emission Tomography for Cartilaginous Tumors of Bone," Journal of Bone and Joint Surgery, Vol. 86-A, No. 12, 2004, pp. 2677-2685.

[11] F. Feldman, R. van Heertum and C. Manos, "18FDG PET Scanning of Benign and Malignant Musculoskeletal Lesions," Skeletal Radiology, Vol. 32, No. 4, 2003, pp. 201208. doi:10.1007/s00256-003-0623-3

[12] F. Feldman, R. Van Heertum, C. Saxena and M. Parisien, "18FDG-PET Applications for Cartilage Neoplasms," Skeletal Radiology, Vol. 34, No. 7, 2005, pp. 367-374. doi:10.1007/s00256-005-0894-y

[13] D. Uesaka, Y. Demura, T. Ishizaki, S. Ameshima, I. Miyamori, M. Sasaki, Y. Fujibayashi and H. Okazawa, "Evaluation of Dual-Time-Point 18F-FDG PET for Stag- 
ing in Patients with Lung Cancer," Journal of Nuclear Medicine, Vol. 49, No. 10, 2008, pp. 1606-1612.

doi:10.2967/jnumed.108.051250

[14] A. Matthies, M. Hickeson, A. Cuchiara and A. Alavi, "Dual Time Point 18F-FDG PET for the Evaluation of Pulmonary Nodules," Journal of Nuclear Medicine, Vol. 43, No. 7, 2002, pp. 871-875.

[15] M. D. Murphey, J. J. Choi, M. J. Kransdorf, D. J. Flemming and F. H. Gannon, "Imaging of Osteochondroma: Variants and Complications with Radiologic-Pathologic Correlation," Radiographics, Vol. 20, No. 5, 2000, pp. 1407-1434.

[16] J. Malghem, B. V. Berg, H. Noel and B. Maldague, "Benign Osteochondromas and Exostotic Chondrosarcomas: Evaluation of Cartilage Cap Thickness by Ultrasound," Skeletal Radiology, Vol. 21, No. 1, 1992, pp. 33-37.

\section{doi:10.1007/BF00243091}

[17] T. M. Hudson, D. S. Springfield, S. S. Spanier, W. F. Enneking and D. J. Hamlin, "Benign Exostoses and Exostotic Chondrosarcomas: Evaluation of Cartilage Thickness by CT," Radiology, Vol. 152, No. 3, 1984, pp. 595-599.

[18] M. J. Geirnaerdt, P. C. Hogendoorn, J. L. Bloem, A. H. Taminiau and H. J. van der Woude, "Cartilaginous Tumors: Fast Contrast-Enhanced MR Imaging," Radiology, Vol. 214, No. 2, 2000, pp. 539-546.

[19] S. A. Bernard, M. D. Murphey, D. J. Flemming and M. J. Kransdorf, "Improved Differentiation of Benign Osteochondromas from Secondary Chondrosarcomas with Standardized Measurement of Cartilage Cap at CT and MR Imaging," Radiology, Vol. 255, No. 3, 2010, pp. 857-865. doi:10.1148/radiol.10082120 